This item was submitted to Loughborough's Research Repository by the author.

Items in Figshare are protected by copyright, with all rights reserved, unless otherwise indicated.

\title{
Influence of haematocrit level on the kinetics of blood spreading on thin porous medium during dried blood spot sampling
}

PLEASE CITE THE PUBLISHED VERSION

http://dx.doi.org/10.1016/j.colsurfa.2014.03.033

\section{PUBLISHER}

(C) Elsevier

\section{VERSION}

AM (Accepted Manuscript)

\section{LICENCE}

CC BY-NC-ND 4.0

\section{REPOSITORY RECORD}

Chao, Tzu-Chieh, Anna Trybala, Victor Starov, and Diganta Bhusan Das. 2014. "Influence of Haematocrit Level on the Kinetics of Blood Spreading on Thin Porous Medium During Dried Blood Spot Sampling". figshare. https://hdl.handle.net/2134/14335. 
This item was submitted to Loughborough's Institutional Repository (https://dspace.lboro.ac.uk/) by the author and is made available under the following Creative Commons Licence conditions.

\section{creative
commons}

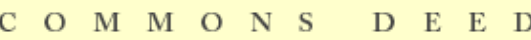

Attribution-NonCommercial-NoDerivs 2.5

You are free:

- to copy, distribute, display, and perform the work

Under the following conditions:

Attribution. You must attribute the work in the manner specified b the author or licensor.

Noncommercial. You may not use this work for commercial purposes.

No Derivative Works. You may not alter, transform, or build upon this work.

- For any reuse or distribution, you must make clear to others the license terms of this work.

- Any of these conditions can be waived if you get permission from the copyright holder.

Your fair use and other rights are in no way affected by the above.

This is a human-readable summary of the Leqal Code (the full license).

\section{Disclaimer 만}

For the full text of this licence, please go to: http://creativecommons.org/licenses/by-nc-nd/2.5/ 


\title{
Influence of haematocrit level on the kinetics of blood spreading on thin porous medium during dried blood spot sampling
}

\author{
Tzu Chieh Chao, Anna Trybala, Victor Starov, Diganta B. Das*
}

Department of Chemical Engineering, Loughborough University, Loughborough, LE11 3TU, UK

( ${ }^{*}$ Corresponding author; Email: D.B.Das@lboro.ac.uk, Tel: 00441509 222509)

\section{Abstract}

Dried blood spotting (DBS) is a convenient blood collecting and sampling method which is widely applied in newborn screening and blood analysis. At the moment, the practice is to try to keep the blood within a marked circle in a thin porous filter paper. However, it is not always possible to predict exactly how the blood spot spreads inside the filter papers and it depends on many factors including the properties of the filter papers, blood properties and how the blood is deposited on the filter paper. In this paper, we aim to identify the relationships between the physical properties and the spreading behaviour of blood on a typical DBS filter paper (Whatman 903). Pig's blood was used to mimic the behaviour of human blood and investigate the spreading/imbibition processes of blood drops on the filter paper. Both top and side views were used to analyse the spreading/imbibition behaviour. The experimental data present the haematocrit effect on the spreading dynamics of blood for dried blood spot sampling. The results obtained prove that the spreading/imbibition time dependences of droplet height, droplet base radius and contact angle are universal function of dimensionless time.

Keywords: Dried blood spots (DBS), spreading dynamics, spreading experiment, dynamic contact angle, haematocrit level

\section{Introduction}

Dried blood spotting (DBS) is a convenient blood collecting and sampling method, which is widely applied in newborn screening and blood analysis. The ease of its use and a number of other benefits derived from advanced analytic technology have led to a rapid growth in the application of DBS in traditional screening methods (e.g., large scale neonatal screening) and others, e.g., preclinical test and, pharmacokinetic (PK), toxicokinetic (TK) and therapeutic drug monitoring (TDM) [1-8]. DBS provides many benefits compared to conventional whole blood collection or plasma sampling, such as low cost, ease of transport and storage, etc. [9]. These benefits are derived from the ability of DBS to collect, handle and store blood samples of microvolumes from which qualitative and quantitative data can be obtained at a later date $[1,2,10]$. However, this method suffers from two main problems. First, the dispersion of blood analytes over filter paper is often found to be unequal which may cause inaccuracies in the clinical analysis of the collected blood [11]. Second, the current DBS methods may not be applied to 
analyse certain analytes due to the small sampling volume and low recovery. The latter problem has been benefitted from advanced analytical methods recently, such as liquid chromatography tandem mass spectrometry (LC-MSMS) and high performance liquid chromatography-ultraviolet (HPLC-UV) $[1,2,6,10]$. However, the issues mentioned above have always been the bottleneck of practical application of DBS.

According to a number of review papers $[3,6,7,10,12,13]$, most researches of DBS are focussed on the metabolic disorder and clinical disease analyses, and studies on their fluid dynamical behaviour (e.g., spreading kinetics of blood above and within the filter paper) are much less visible [5-8]. At the moment, the practice is to try to keep the blood samples within a marked circle in a thin porous filter paper. Recently, a significant amount of work has been spent trying to find out how much the blood spot will spread (spreading behaviour) without trying to quantify the kinetics of the wetting properties of the filter paper $[2,9,11,12,14,15]$. Also, a large amount of the work seems to have been spent on trying to develop techniques for measuring concentrations of solute/molecules from the collected blood sample on filter papers $[2,7,10,12]$. However, it is well known that the spreading behaviour of blood droplets are not always possible to predict exactly on the filter papers and it depends on many factors including the properties of the filter papers, blood properties and the way how blood is deposited on the filter paper. Therefore, there is no well-defined relationship between the theoretical and experimental parameters in the published DBS literature and only few researches have considered the influence of spreading dynamics on the DBS without quantifying these behaviour in detail $[3,9,16]$. In order to understand the spreading processes accurately new model based on both theoretical and experimental methods should be developed since the study of spreading behaviour of the blood drop over DBS filter paper had not been developed before. In addressing these points, the spreading and imbibition of blood droplets on thin porous media, namely, DBS filter paper is studied in this paper.

The kinetics of the spreading of other liquid drops over porous medium has been investigated in previous studies [17-19]. According to the previous studies, the drop spreading over dry porous layers is considered as two competitive processes: (i) the spreading of the drop over an saturated porous surface and (ii) the imbibition of the liquid from the drop into the porous substrate [20]. In this paper an axisymmetric experimental model of liquid drop spreading over a thin porous layer is adopted as discussed in the next section. In the experiments, the dynamic contact angles, droplet base radius and profile have been measured to characterize the spreading process. 
Although some of the above studies reported the spreading/imbibition of droplets of Newtonian liquids on thin porous media, there is little or no study that has reported the spreading behaviour of non-Newtonian fluids in general and, more specifically, blood in the context of DBS. Therefore, the experimental investigation on blood spreading behaviour is essential. As mentioned earlier, there are a number of parameters which affect the spreading behaviours of blood on filter papers. Firstly, the physical properties of a filter paper, such as an average pore size and, thickness on the layer affect the capacity and spreadability of blood on the filter paper. Considering the consistency of the properties of DBS filter papers, the performance of filter paper was monitored by the Newborn Screening Quality Assurance Program (NSQAP) at the Centers for Disease Control and Prevention (CDC) (Atlanta, USA) to ensure that new filter paper are consistent with established guideline [21]. Secondly, the properties of blood, including, blood rheology, haematocrit level (i.e., the volume fraction of red blood cells in blood) and drop volume affect the performance of blood spreading/imbibition.

It is well known that the blood rheology is affected by the haematocrit level $[22,23]$. Further, the significance of haematocrit level to dried blood sampling has been discussed earlier $[9,11,24-26]$. For example, it was reported that the levels of most amino acids and free carnitine were higher in the blood drop periphery than in the central spot with lower haematocrit level [11]. Denniff and Spooner [9] reported that a bias was observed in the concentration of two analytes at different haematocrit levels and the area of DBS samples decreased linearly with increasing haematocrit levels. [9]. O'Mara et al. [24] reported that a significant bias (>15\%) existed due to the haematocrit effects and unequal distribution across the spot. This shows that the influence of haematocrit levels on the concentration of analytes would be case-dependent, i.e., analyte concentration could vary in different cases of the DBS samples, which could be caused by unequal distribution of analytes in plasma, red blood cells or both [25].. In order to utilize DBS accurately in clinical analyses, the haematocrit effects should be investigated as a method development and validation for individual analyte. Generally speaking, it is expected that the influence of the spreading kinetics of blood droplet at different haematocrit level to the DBS sampling is much more consistent although the spreading kinetics is determined by the rheology of blood, which again depends on haematocrit level. Nevertheless, the spreading kinetics of blood at different haematocrit levels should be investigated further to provide a better consideration of the influence of haematocrit level differences to the whole DBS sampling and analysis process.

105 In addressing the above issues, a series of experiments is presented in this paper to investigate 106 the spreading/imbibition behaviour of blood droplets with different haematocrit levels on DBS 
filter papers. The experiments are aimed at recording blood drop spreading/imbibition behaviour over the filter paper using a high speed camera and analysing the spreading droplet radius, volume, wetted area inside the filter paper and contact angle by an image analytic software $[17,19,27]$. The whole process requires special conditions in which they are carried out as the spreading/imbibition experiment data may be influenced by environmental factors such as, gas flow, vibration and horizontal level. Therefore, in our experiment, a special hermetically isolated chamber has been designed and installed on a vibration-protected table to eliminate the environmental effects during the drop spreading experiments. In order to quantify the blood spreading process, we observe the wetted region on the surface of filter paper (top view) as well as the droplet spreading and absorption behaviour (side view). The wetting region on the surface of the filter paper is known as the dried blood spots sample area. According to Starov et al. [19], the spreading behaviour of liquid droplet over porous layer (filter paper in our case) could be considered as overlapping of two different processes: one is the spreading of blood over the filter paper; another is the capillary motion inside the matrix of the filter paper. In consistent with this study, the time evolution of the radius of the wetting region, the drop base, the drop volume and the contact angle were monitored in our experiments.

We use pig's blood with different haematocrit levels to simulate the behaviour of human blood, and observe the spreading process of the liquid drops on DBS filter paper to analyse its spreading behaviour. The selection of the animal blood as a simulant for human blood in our experiments is based on the similarity of its rheological properties to those of human blood, namely, the blood viscosity, plasma viscosity, erythrocyte aggregation and others [28]. The easy availability of pig's blood from nearby abattoir and the ethical policy on minimum or no use of human blood for laboratory experiments are other considerations in our design of experiments. Accordingly, pig's blood is considered as the most suitable blood simulant in our experiments. The blood rheology was obtained via in-house laboratory experiments and used to identify the difference of spreading behaviour between blood samples with different haematocrit levels. The spreading behaviours of blood plasma and water on the same filter paper are also analysed as reference liquid and used to characterise the effects of the presence of red blood cells (RBCs) to the spreading behaviour of pure liquid.

\section{Materials and Experimental Methods}

\subsection{Blood}

140 Pig's blood was collected in EDTA anti-coagulated tubes (International Scientific Supplies Ltd. 141 Bradford, UK) from a local butcher. 0.9\% sterile saline solution (OXOID Ltd., Hampshire, UK) 
142 was used to wash red blood cells after centrifugation. Blood was stored at $4^{\circ} \mathrm{C}$ and all blood

143 samples were used within 4 hours after collection.

\section{$144 \quad$ 2.1.1 Preparation of different haematocrit levels of blood}

145 Blood of different haematocrit levels was prepared according to the procedures presented by 146 Baskurt et al. [23]. The blood samples with different haematocrit levels were centrifuged by a 147 Heraeus Labofuge 400R centrifuge (Thermo scientific, UK) at a constant rotating speed ( 1400 g) 148 for 10 minutes to separate the red blood cells (RBC) and plasma without damaging the RBC in 149 the blood. After the separation, the plasma was kept for re-suspension later and the cells were 150 washed by the sterile saline solution three times to remove buffy coat layer, i.e., a thin layer that 151 is generated between plasma and RBC after centrifugation, which also contains most of the 152 white blood cells and platelets. The washed cells were then re-suspended in plasma at the 153 required haematocrit levels, namely, $0 \%, 30 \%, 50 \%$ and $70 \%$.

After the preparation of blood samples, the haematocrit level and cell density were measured for every sample to make sure consistency in the quality of the samples.

\subsubsection{Measurements of blood rheology}

159 As described in section 2.1.1, blood samples with different haematocrit levels $(0 \%, 30 \%, 50 \%$, 160 and $70 \%$ ) were prepared from single source and slowly vibrated to a well-mixed condition at room temperature before testing. The blood rheology measurements have been made using the rheometer with plane geometry $(4 \mathrm{~cm}$ diameter, stainless steel) and $250 \mu \mathrm{m}$ gap. The temperature was kept constant using Peltier plate at $25^{\circ} \mathrm{C}$. The viscosity measurements have been made in shear rate range 0.2 to $100 \mathrm{~s}^{-1}$. Also the blood density was measured using a pycnometer at $25^{\circ} \mathrm{C}$.

\subsection{Filter paper characteristic}

167 Whatman 903 blood spot cards were supplied by Whatman (GE Healthcare, Maidstone, UK).

168 Riechert-Jung MEF3 inverted microscope accompanied with the digital image acquisition via 169 QCapture software are used to estimate the filter paper thickness. Carl Zeiss (Leo Cambridge) 170 Stereoscan 360 Scanning electron microscopy (SEM) was applied to provide the scanning 171 electron micrographs which are then used to determine the thickness, the surface porosity and 172 the pore size distribution. All the experimental results for filter paper thickness are calculated 173 from SEM and inverted optical microscope images via Image $\mathrm{J}$ software.

\section{2.3. Spreading experiment}

175 The spreading of blood with different haematocrit levels was observed from both side and top. 176 Each spreading data were determined from ten replicate blood spot, each derived from $10 \mu \mathrm{l}$ 
blood samples, at $0 \%, 30 \%, 50 \%$ and $70 \%$ haematocrit levels on DBS filter paper. Each droplet sample was produced via a $10.0 \pm 0.5 \mu \mathrm{l}$ syringe in our experiments. Furthermore, the initial volumes of the blood droplets were calculated and checked from images recorded by high speed camera for all the spreading experiments. Hence, the experiments conducted in this work ensure repeatability of initial droplet volume within a range of around $10.0 \pm 0.5 \mu \mathrm{l}$. All the video images were taken from printed side of DBS filter paper with a constant working distance and focal length.

Cameras that have been used in spreading experiment are AVT Pike F-032 high performance camera (Allied Vision Technologies, UK) for the top views recording and i-SPEED LT high speed video camera (Olympus, UK) for the side views. The optical objective used in side view experiment for small drop spreading is IF-3 standard (INFINITY PHOTO-OPTICAL GmbH, Germany). AR1000-N Rheometer (TA instrument, USA) was used to determine the viscosity of blood.

The experimental set up of spreading experiment is shown in Figure 1, which is based on the experiment setup used earlier for the droplet spreading/imbibition experiments with Newtonian liquids [20]. A filter paper substrate is placed in a closed chamber with a fixed temperature and humidity. The chamber was equipped with optical glass windows for observation of both the shape and the size of the spreading drops; a side view and top view were monitored. The light source was installed at the bottom and the other side of chamber where the light can come from the opposite site of the CCD camera. The chamber and all optical equipment were mounted on an optical bench. The optical bench was installed on a vibration-protected table.

The experiments were processed in the following order for obtaining both side and top views of blood spreading:

203

a) The porous substrate under investigation (filter paper) was placed in the chamber.

b) Optical equipment was adjusted to focus on the sample depositing spot and the spreading process area, where the equipment include: CCD camera lenses, light source based on working distance.

c) A droplet of investigated sample (blood) was placed on the substrate by syringe and the whole process was recorded by CCD cameras.

d) Calibration of known distance in each image was done with identified micro-scale. 
e) The image was analysed to obtain experimental data via image processing software (ImageJ and Vision builder (National Instrument, UK)) which are able to record specific parameters such as droplet radius, height and wetting region and quantify the obtained data.

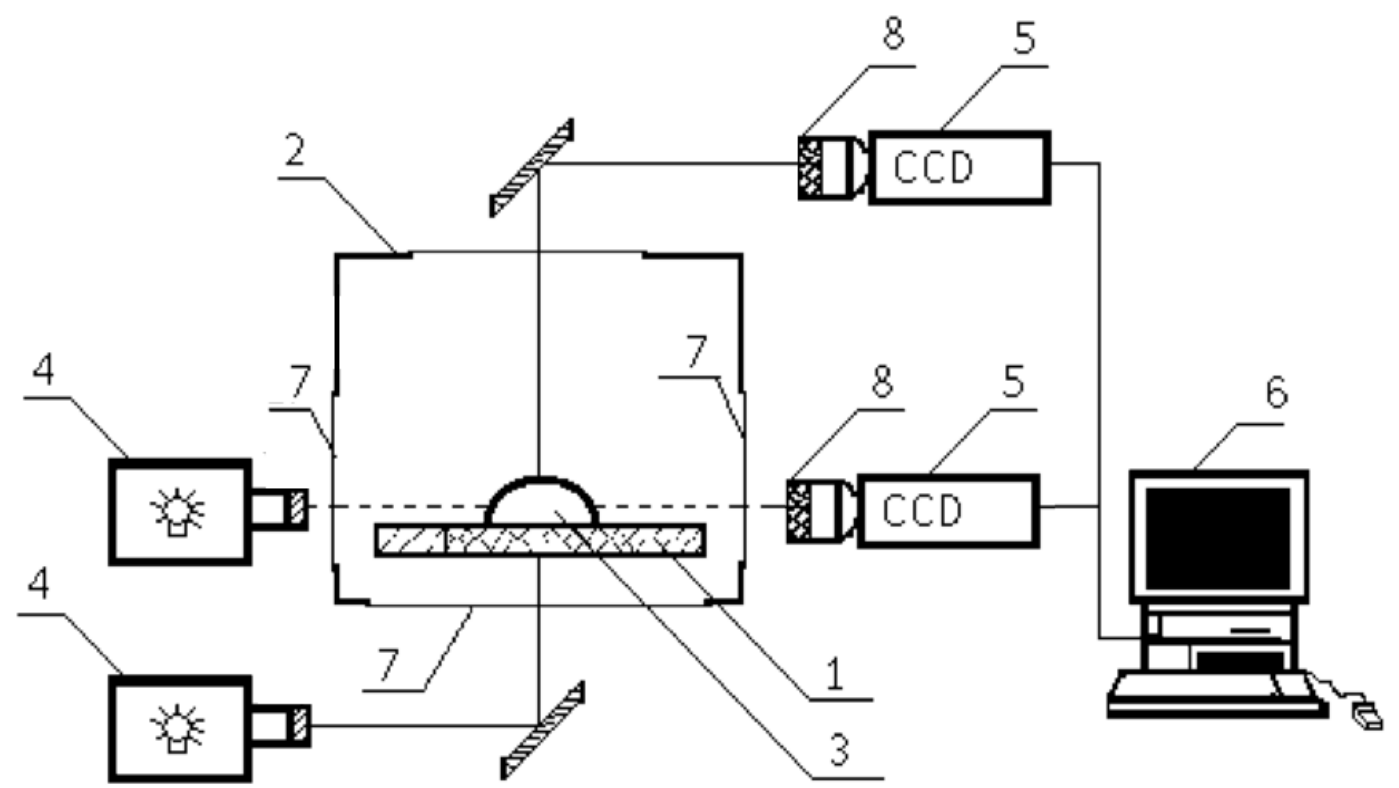

215

216

Figure 1. Sketch of the experimental set-up: 1 , porous substrate; 2 , hermetically closed, thermostated chamber, 3, liquid drop; 4, light sources; 5, CCD cameras; 6, PC; 7, optical windows; 8, optical objectives.

\section{Results and Discussions}

\subsection{Rheology of blood used in spreading experiments}

The rheology of blood is an important parameter in the spreading dynamics over porous medium. The influence of haematocrit level of blood viscosity was investigated by measuring the blood viscosity at different haematocrit levels. In particular they were measured so as to characterise the blood samples used in this study. The viscosity values were measured at $25^{\circ} \mathrm{C}$, which are shown in Figure 2. As expected, the shear thinning character of the blood was found. At high shear rate, the value of viscosity of blood decreases to a constant low number of around 4-15 cP. However, at low shear rate the blood viscosity varies from $1000 \mathrm{cP}$ to $400 \mathrm{cP}$ which may be caused by the unequal aggregation of RBCs and the formation of suspended particles inside the blood [23]. Figure 2 shows that the viscosity of blood is higher as the haematocrit level increases. The experimental data presented in the figure are in a good agreement with other reference data $[28,29]$. The data are also in good agreement with Thiriet's study [30] in which the author shows that the values of blood viscosity at constant temperature can be approximated by following power law equation: 
where $\mathrm{k}$ and $\mathrm{n}$ are consistency factor and flow behaviour index, $\mu$ is blood viscosity and $\dot{\gamma}$ is the shear rate. Equation (1) can be rewritten as $\ln \mu=\ln k-n \ln \dot{\gamma}$ and we use Table 1 to fit the parameters $\mathrm{k}$ and $\mathrm{n}$.

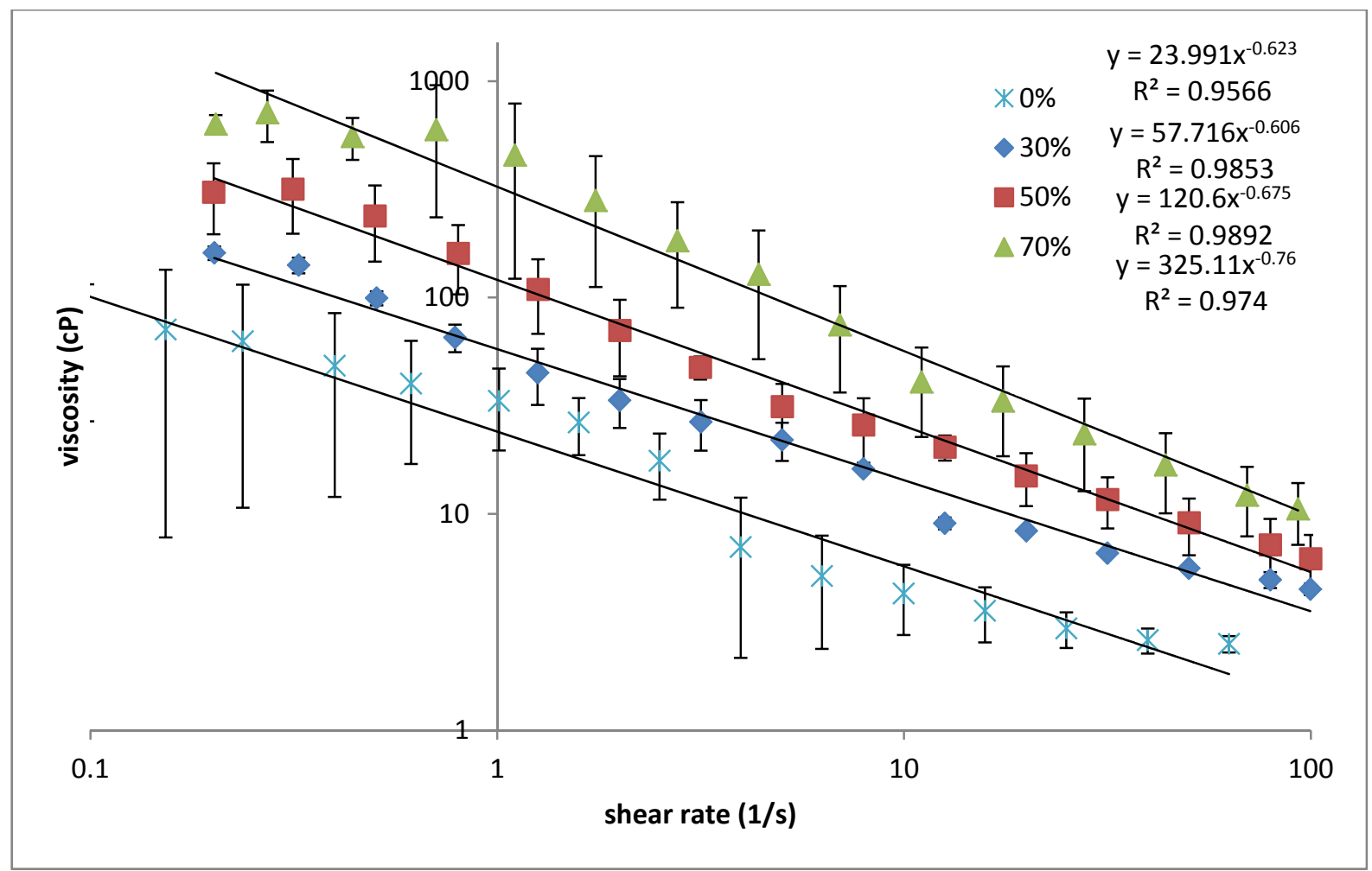

Figure 2. The dependancy of blood viscosity at $0 \%, 30 \%, 50 \%$ and $70 \%$ haematocrit levels on shear rate from $0.5 \mathrm{~s}^{-1}$ to $100.0 \mathrm{~s}^{-1}$

Table 1. Fitted values of $\mathrm{k}$ and $\mathrm{n}$ according to equation (1)

\begin{tabular}{|l|l|l|}
\hline Haematocrit levels in blood & $\mathrm{k}$ & $\mathrm{n}$ \\
\hline $0 \%$ & 23.99 & 0.368 \\
\hline $30 \%$ & 57.72 & 0.394 \\
\hline $50 \%$ & 120.60 & 0.325 \\
\hline $70 \%$ & 325.11 & 0.240 \\
\hline
\end{tabular}

As shown in Table 1, the values of $\mathrm{n}$ are almost constant and it is slightly higher with higher haematocrit level. However, the values of k vary significantly with different haematocrit levels.

247 These show that the viscosity values of blood follow a universal function of shear rate, where the 248 values increase with increasing haematocrit levels. 
The average densities of the blood sample used in this study are shown in Table 2. The results show that the density of blood sample with higher haematocrit level is higher than blood samples with lesser haematocrit level.

Table 2. The average density of plasma and blood at 30\%, 50\% and $70 \%$ haematocrit levels at $25^{\circ} \mathrm{C}$ (data were calculated based on 3 samples)

\begin{tabular}{|l|r|}
\hline Fluid Sample & Average Density $\left(\mathbf{k g} / \mathbf{m}^{3}\right)$ \\
\hline Plasma (0\% haematocrit level in pig's blood) & $1030.52 \pm 0.13$ \\
\hline $30 \%$ haematocrit level in pig's blood & $1043.18 \pm 1.32$ \\
\hline $50 \%$ haematocrit level in pig's blood & $1049.75 \pm 1.75$ \\
\hline $70 \%$ haematocrit level in pig's blood & $1058.71 \pm 2.21$ \\
\hline
\end{tabular}

\subsection{Characteristics of filter paper}

258 The spreading/imbibition behaviour of blood droplet over porous filter paper depends on the 259 physical properties of filter paper used: the thickness, fibre matrix composition, permeability, 260 porosity and pores size distribution. We used two microscopes to observe the surface heterogeneity and measure the thickness of filter paper, in order to analyse these physical parameters under different scale levels (Figure 3). In Figure 3, the images have clearly shown that the pores inside the filter paper are created from both the space between the fibre structure and the micro pores of fibres itself. The thickness of filter paper was also measured from the cross sectional images of the filter paper which were taken by an inverted microscopy. The average thickness data were obtained and calculated from 5 random cross sections per image via an image processing software, namely, QCapture (Qimaging, Canada). The image taken by polarized optical microscopy shows that the average thickness of the filter paper is around $\Delta=500$ $\pm 50 \mu \mathrm{m}$, which is a good agreement with data calculated from 10 different samples using direct measurement of the thickness by a micrometre. The intrinsic permeability of the completely wet filter paper was measured using a porometer (Porous Materials Inc., US) which was found to be $0.56 \mp 0.025 \mu \mathrm{m}^{2}$. The basic principle of porometer test is that it forces a non-reactive gas (air in this study) flow through the porous sample with increasing pressure steps to push out the wetting liquid inside porous material. This provides a relationship between the flow rate and pressure difference which is then used to determine the permeability (i.e., Darcy law)[31] 

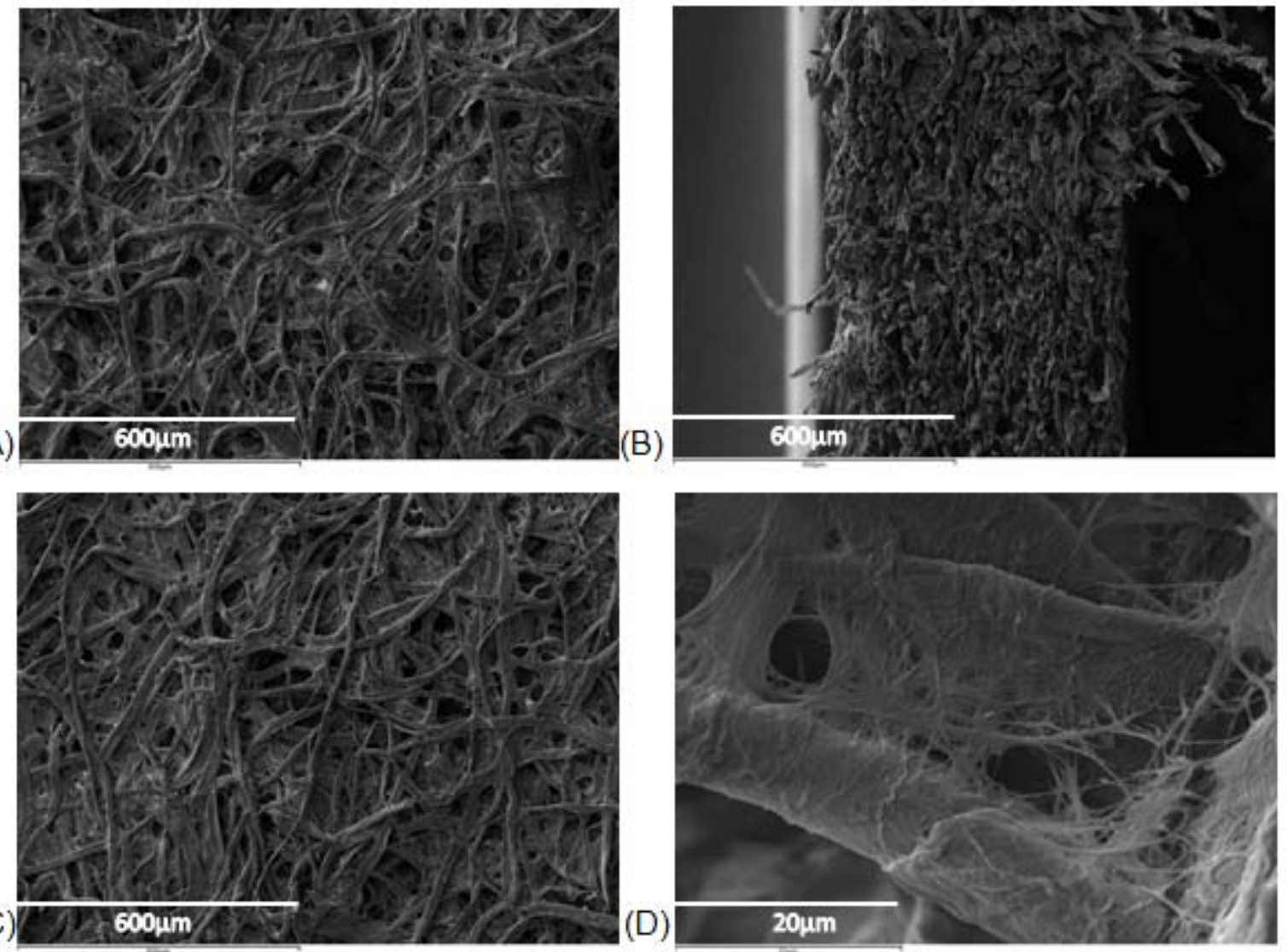

Figure 3. The micrographs of filter paper at (A) top, (B) side and (C) bottom sides under $600 \mu \mathrm{m}$ scale and (D) $20 \mu \mathrm{m}$ scale from scanning electron microscopy (SEM)

\subsection{Kinetics of blood droplet spreading}

280 The images captured from top and side views were analysed for all spreading/imbibition experiments. The drop spreading/imbibition were assumed to be symmetric over the whole duration of the process and the contact area of the drop on the surface of the porous filter paper was assumed to be circular. These assumptions were in a good agreement with our experimental observations of blood spreading/imbibition processes. Spherical form of the spreading drop was used to calculate the volume of the drop that lies over the substrate as a function of time. The schematic is shown in Figure 4.

The wetted region inside the filter paper, $\mathrm{l}(\mathrm{t})$, was obtained using top view video photographs at $60 \mathrm{fps}$ (Figure 5). These image sequences were processed using ImageJ software to obtain the area $A(t)$ and perimeter $P(t)$ of each spot. $A$ distance was defined on the image of an identified micro-scale using the software calibration; this was then used to measure the area of the DBS samples after transforming the image into black and white. The hydraulic radius of wetting region I(t) was then calculated as follows: 
297

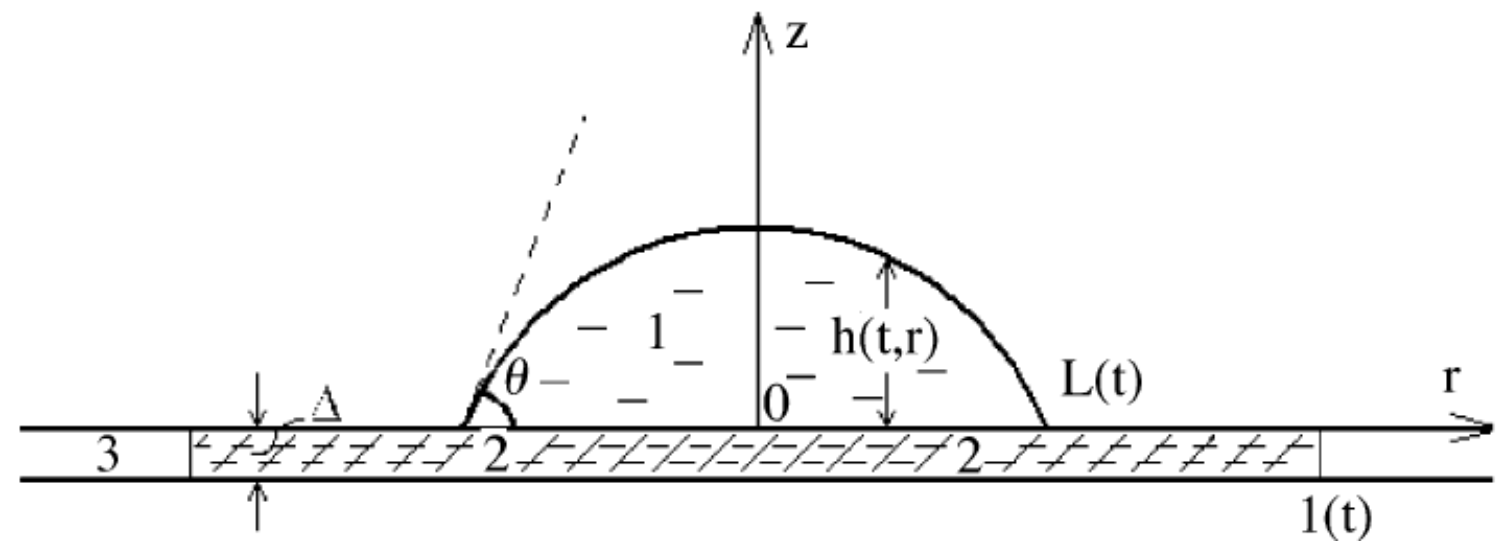

Figure 4. Cross-section of the axis-symmetric spreading drop over initially dry filter paper with thickness $\Delta$. 1 -liquid drop; 2 - wetted region inside the porous substrate; 3 - dry region inside the porous substrate. $L(t)$ - radius of the drop base; I $(t)$ - radius of the wetted area inside the porous substrate; $\Delta$-thickness of porous substrate, $r, z$ co-ordinate system; $h(t, r)$-profile of the spreading drop; $\boldsymbol{\theta}$-contact angle.

The time dependency of the radii of wetting regions, $I(t)$, for pure water and blood at $0 \%, 30 \%$, $50 \%$ and $70 \%$ haematocrit levels on Whatman 903 filter paper at $25^{\circ} \mathrm{C}$ are shown in Figure 6. Figure 6 shows that the spreading process inside the filter paper can be divided into two stages in the case of pure water and plasma ( $0 \%$ haematocrit blood). The first stage of the penetration of fluid inside the filter paper is caused by the imbibition of the blood from the droplet transport into big pores. As a result the wetted region increased very fast. After the imbibition of droplet was completed, the second stage involves the penetration of fluid inside the filter paper, which is caused by the fluid penetration from larger pores into smaller pores, where the spreading kinetic is much slower.

According to Figure 6 there is a significant difference in the radius of wetted region in the case of (1) the water (Newtonian liquid) and (2) plasma and blood samples (shear thinning liquids). First of all, the radius of wetting region of water is much bigger than in the case of plasma and blood samples. In the case of plasma/blood samples, the radius of the wetted region reached its maximum value within around 0.4 second and stopped expanding further. On the other hand, the water drops spread continuously for a prolonged period of time after the droplet spreading was completed. Water continued to spread inside the filter paper after the droplet on the filter paper already disappeared. However, all the plasma/blood samples stopped penetrating around the same time when the droplet was completely imbibed into filter paper. These results indicate that the presence of RBCs change considerably the spreading behaviour of blood inside the filter 

to penetrate inside the filter paper.

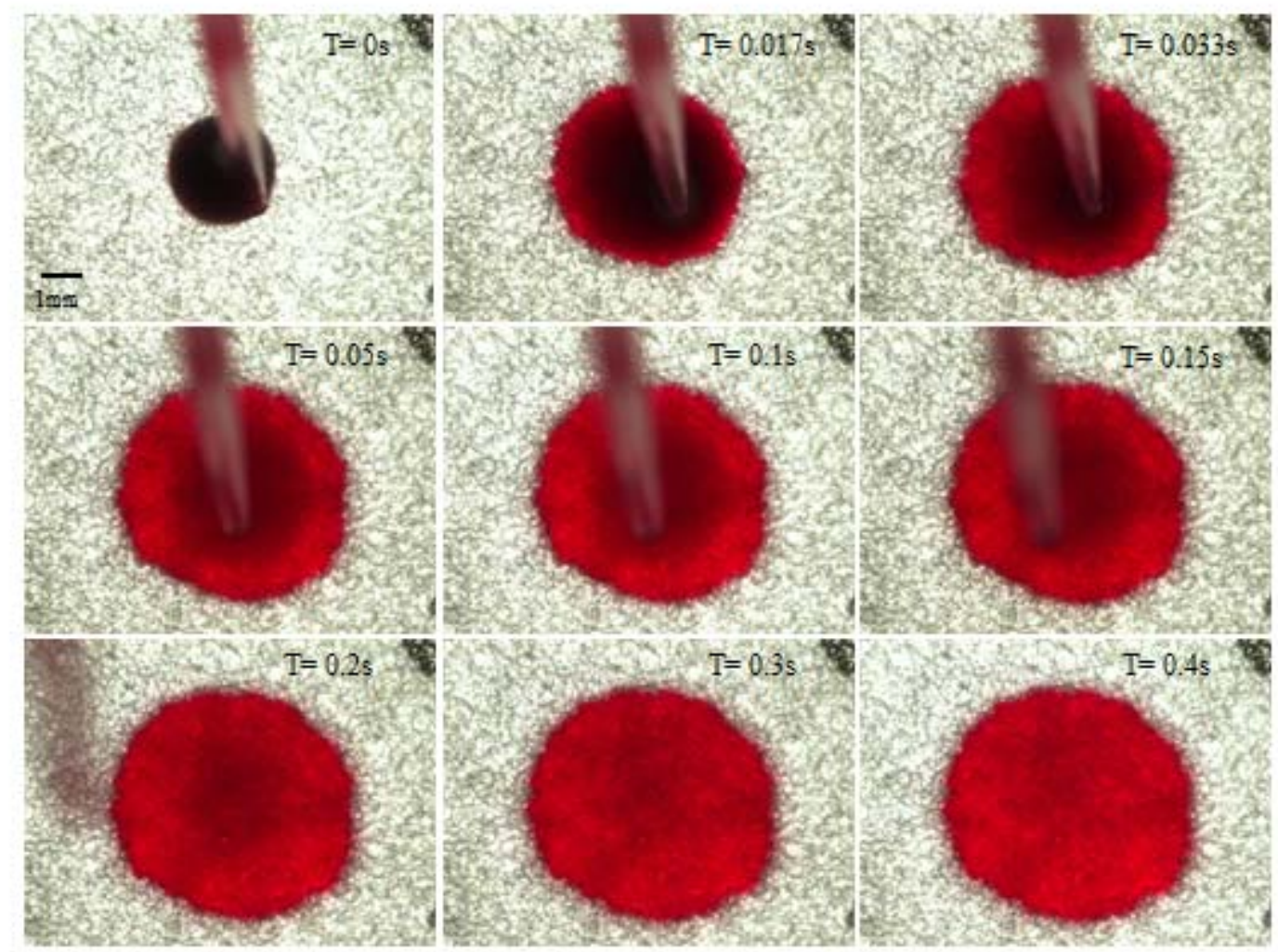

326

327

328

329

330

331

332

333

334

335

336

337

338

339

340

341
Figure 5. Top views of $30 \%$ haematocrit blood spreading on filter paper. Each image was captured at $60 \mathrm{fps}$ from the time the first image was taken

According to Starov et al. [19], the main mechanisms of spreading behaviour of a liquid drop inside and over the porous substrate are viscosity and capillary forces. The capillary forces do not change a lot with the haematocrit difference as the fluid properties are almost same in the interfacial. Therefore, the viscosity difference between plasma and blood should be the major mechanism to be responsible for the spreading behaviour inside the porous fibre. According to our measurements (Figure 2) both plasma and blood show a shear thinning behaviour. After the blood is imbibed into filter paper, the shear rate of blood decreased and the latter results in the increasing of blood viscosity, which further decrease the spreading kinetic inside the filter paper and eventually prevent the further penetration of blood. In physical terms the latter means that the red blood cells block the micro-pores in the filter paper and as a result the plasma cannot penetrate from the large pores into smaller pores. 


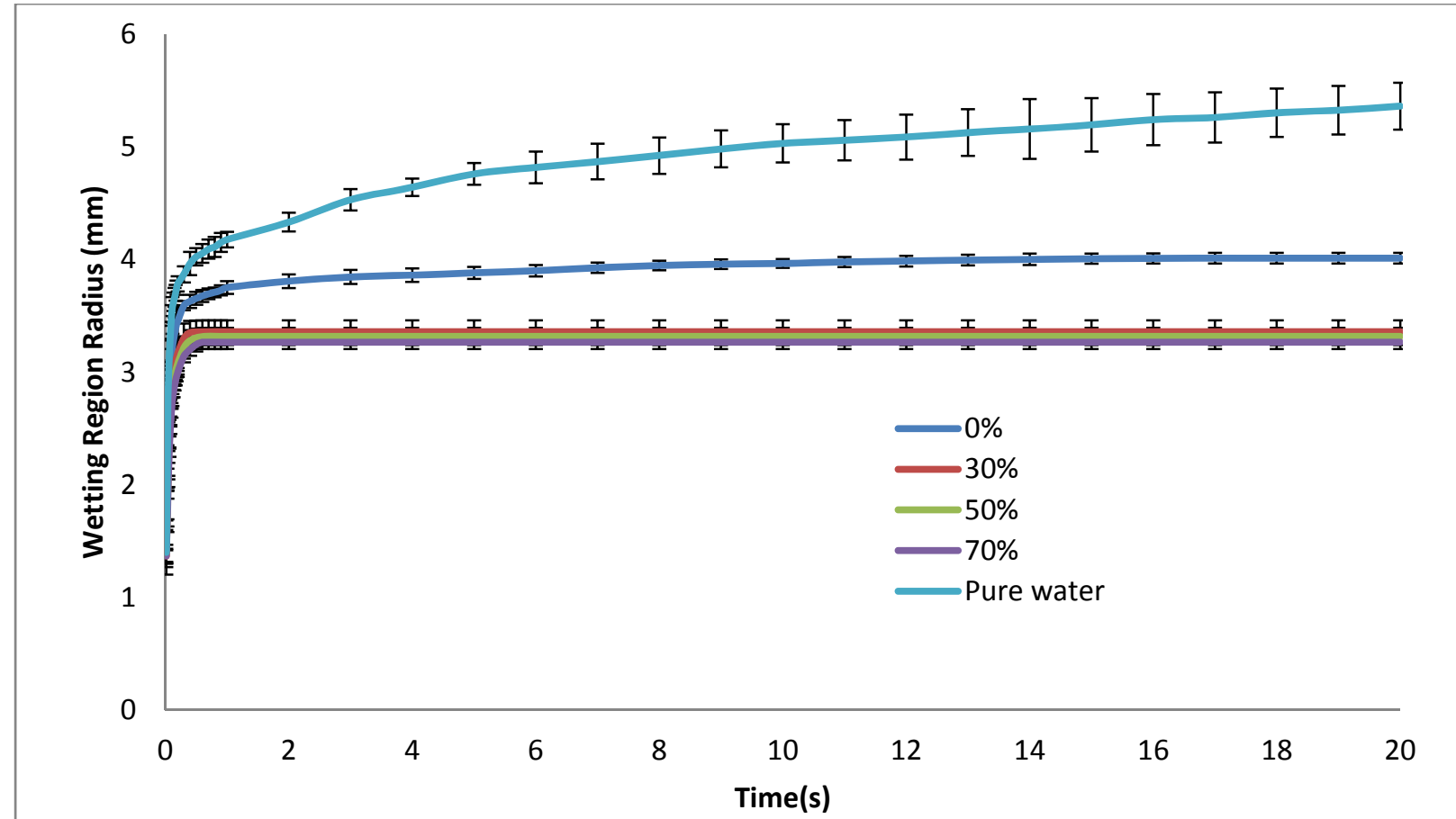

343 Figure 6. Time evolution of radius of wetted region, I $(t)$, inside the filter paper: plasma, pure water and $30 \%, 50 \%, 70 \%$ haematocrit levels in blood

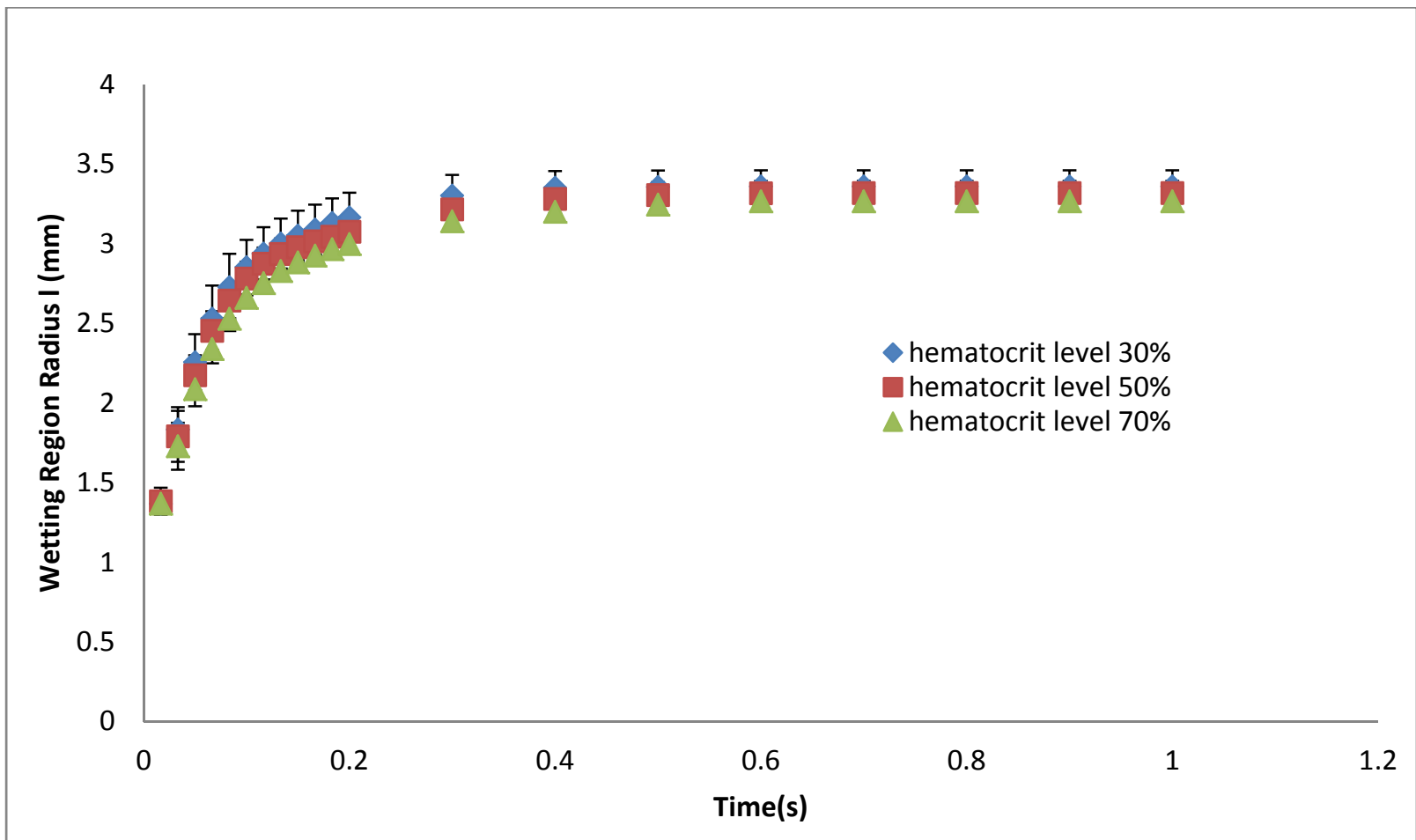

Figure 7. The spreading radii of wetting regions of blood at different haematocrit levels

349 In Figure 7, a magnification of the data presented in Figure 6 is given on the time dependency of 350 the wetted region inside the filter paper for blood at different haematocrit levels. Figure 7 shows 
that the maximum radius of the wetted area slightly decreases with higher haematocrit level: the maximum radius difference between $30 \%$ and $70 \%$ haematocrit blood are around $3 \%$. This result has been noticed earlier [9,11]. Figure 7 also shows that the higher haematocrit level is the slower the spreading is.

The time variation of the drop base radius, $\mathrm{L}(\mathrm{t})$, and the height of drop of the drop, $\mathrm{h}(\mathrm{t}, \mathrm{r})$, were obtained using side view and high speed video camera (600 fps) (Figure 8). The images were processed using vision builder software, where the volume of the spherical cap and the contact angle formed by the fluid with substrate was calculated as follows[20]:

360

$361 \quad r_{c}=\frac{\left(L(t)^{2}+h_{c}^{2}\right)}{2 h_{c}}$

$362 \theta=90-\frac{180}{\pi} \sin ^{-1}\left(\frac{r_{c}-h_{c}}{r_{c}}\right)$,

364 where $h_{c}$ is the height of the drop at central point, $r_{c}$ is the contact radius, and $\theta$ is the contact 365 angle.

According to our experimental observation the radius of drop base initially increases to its maximum value and then slowly decreases till complete imbibition of blood drops. The most notable differences between the samples with different haematocrit levels are the spreading kinetic as shown in Figure 9. The time of completely imbibition is around 0.2 second for plasma. However, the imbibition times for blood samples at $30 \%, 50 \%$ and $70 \%$ are slower and are around $0.3 \mathrm{~s}, 0.45 \mathrm{~s}$ and $0.6 \mathrm{~s}$. This result clearly suggests that the spreading kinetic decreases as the haematocrit level increases or the same as the viscosity increases.

In order to further discuss the spreading dynamics of droplet spreading over porous media, the spreading radius, contact angel and droplet height have been suggested to use the dimensionless unit as follows [19]: $\mathrm{L}(\mathrm{t}) / \mathrm{L}_{\mathrm{m}}, \theta(\mathrm{t}) / \theta_{\mathrm{m}}, \mathrm{h}(\mathrm{t}) / \mathrm{h}_{\mathrm{m}}$ on dimensionless time: $\mathrm{t} / \mathrm{t}^{*}$, where $\mathrm{L}_{\mathrm{m}}$ is the maximum radius of the droplet base; $\theta_{\mathrm{m}}$ and $\mathrm{h}_{\mathrm{m}}(\mathrm{t})$ are the contact angle and droplet height when the spreading radius reaches the maximum value; $t^{*}$ is the time of complete imbibition of the blood droplet. All the relevant data are shown in Table 3. 


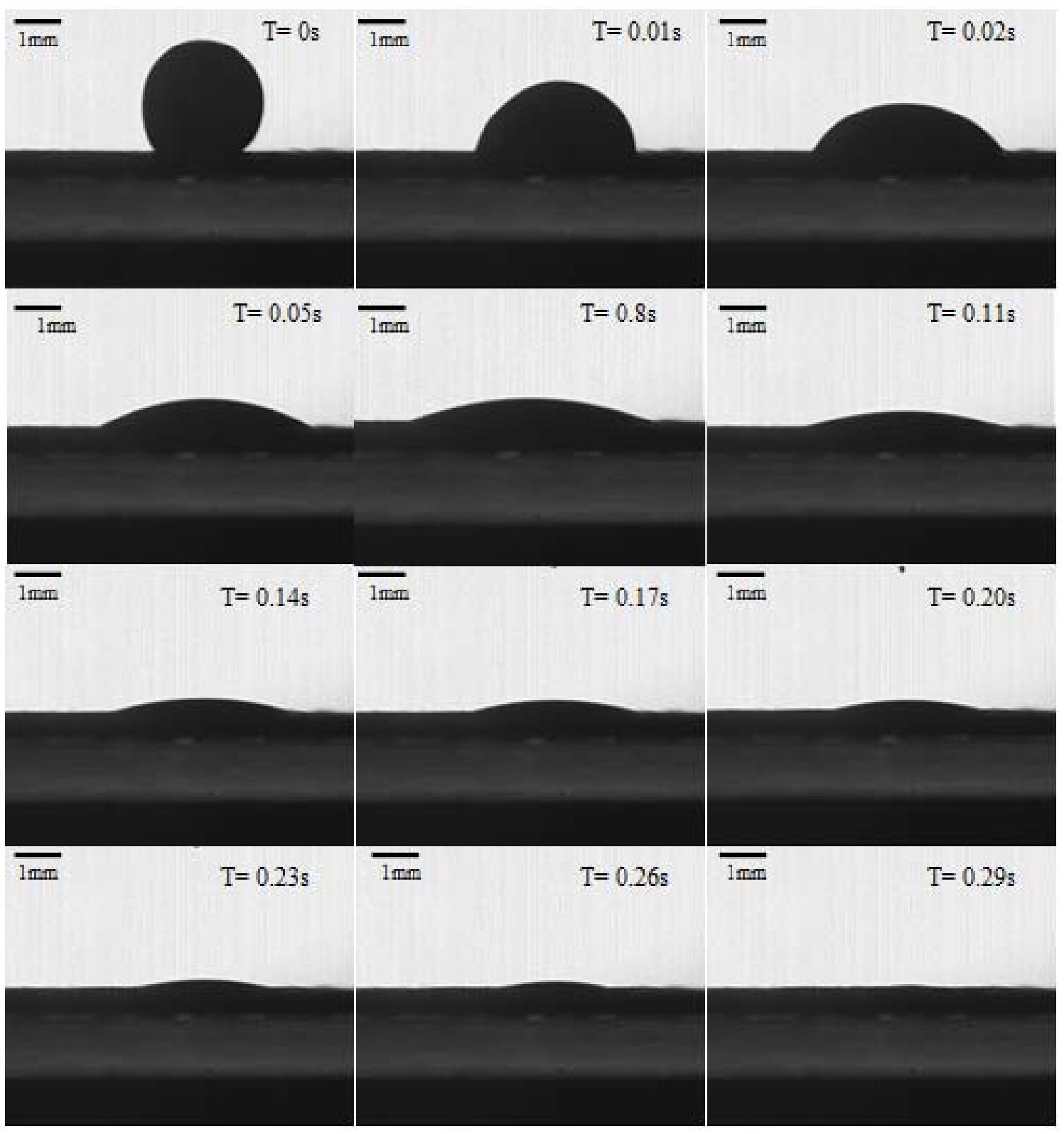

Figure 8 . Side views of $30 \%$ blood spreading over Whatman 903 filter paper. Each image is captured at $600 \mathrm{fps}$ from the time the first image is taken. The time scale for first three pictures is 
Table 3. Time of complete imbibition, maximum spreading radius of droplet base, droplet height at maximum spreading and contact angle at maximum spreading for investigated blood samples.

\begin{tabular}{|l|l|l|l|l|}
\hline & $\begin{array}{l}\mathrm{t}^{*}(\mathrm{~s}) \\
\text { Time of } \\
\text { complete } \\
\text { imbibition }\end{array}$ & $\begin{array}{l}\mathrm{L}_{\mathrm{m}}(\mathrm{mm}) \\
\text { Maximum } \\
\text { spreading radius } \\
\text { of droplet base }\end{array}$ & $\begin{array}{l}\mathrm{h}_{\mathrm{m}}(\mathrm{mm}) \\
\text { Droplet } \\
\text { height at } \\
\text { maximum } \\
\text { spreading }\end{array}$ & $\begin{array}{l}\theta_{\mathrm{m}} \text { (degree) } \\
\text { Contact } \\
\text { angle at } \\
\text { maximum } \\
\text { spreading }\end{array}$ \\
\hline Water & 0.072 & 2.12 & 0.51 & 29.62 \\
\hline $0 \%$ haematocrit level & 0.230 & 2.11 & 0.66 & 34.44 \\
\hline $30 \%$ haematocrit level & 0.351 & 2.18 & 0.65 & 32.80 \\
\hline $50 \%$ haematocrit level & 0.508 & 2.18 & 0.62 & 30.94 \\
\hline $70 \%$ haematocrit level & 0.663 & 2.09 & 0.62 & 33.48 \\
\hline
\end{tabular}

389

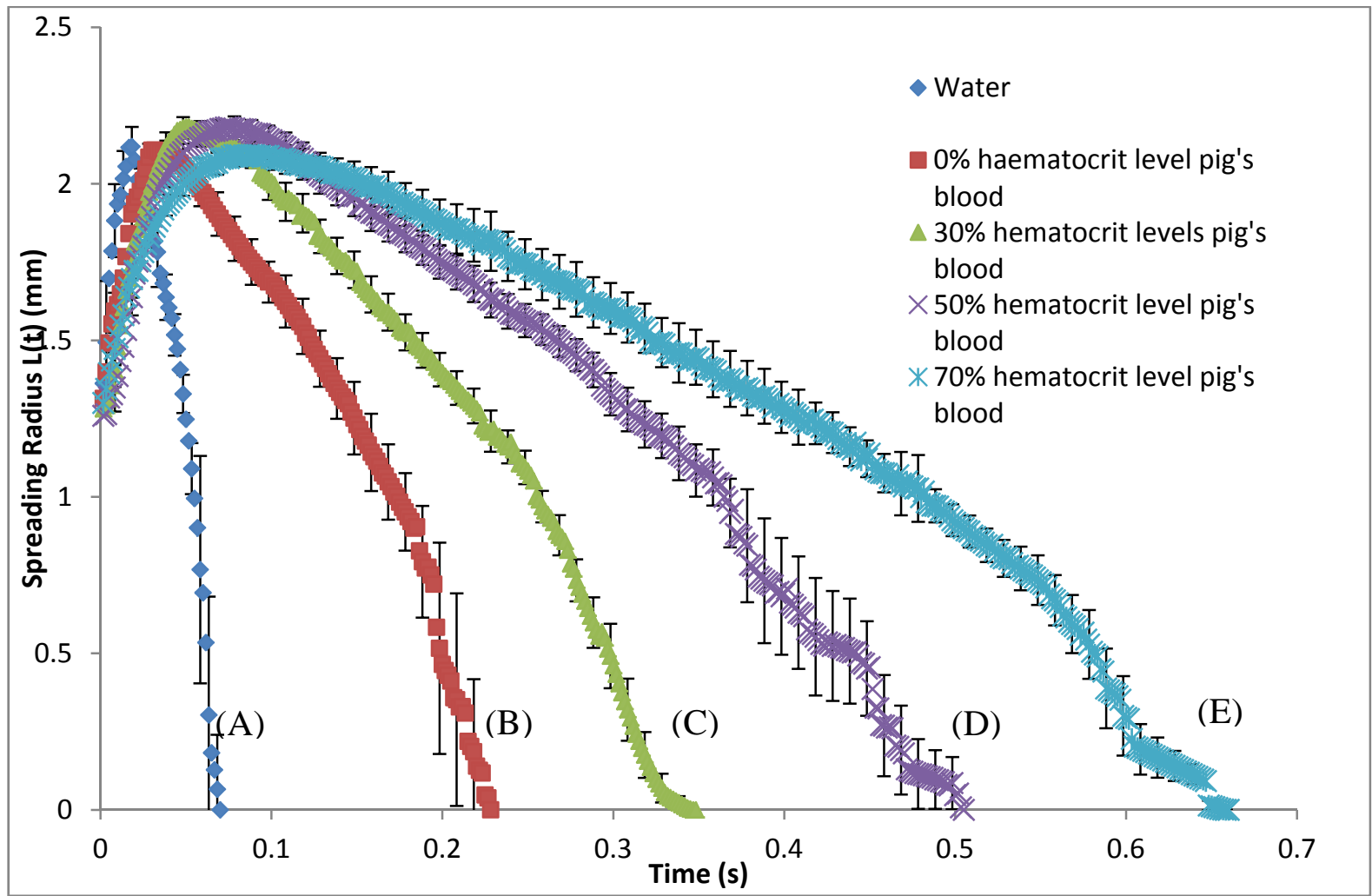

Figure 9. Radii of the drop bases as function time: (A) $10 \mu \mathrm{l}$ water, (B) $10 \mu \mathrm{l} 0 \%$ blood (C) $10 \mu \mathrm{l}$ $30 \%$ blood (D) $10 \mu \mathrm{l} 50 \%$ blood (E) $10 \mu \mathrm{l} 70 \%$ blood on Whatman 903 filter paper.

394 In Figures 10 and 11 the spreading behaviours of pure water and blood with different haematocrit levels in dimensionless units. The dimensionless time scales of blood at different haematocrit levels required to reach the maximum radius of the drop base and completely imbibition are relatively similar compared to water. In reference to the dimensionless time in Figure 10, the 
spreading behaviour of water shows that water has less retention time above the filter paper as compared to that of blood droplets. This indicates that water (a Newtonian liquid) may penetrate easily into the filter paper in comparison to that of the blood samples.

402 Figures 10-12 demonstrate that the time evolution of droplet base, height and contact angle in 403 the case of Newtonian liquid (water) is different from the corresponding dependences of non404 Newtonian liquids: blood droplet at different haematocrit levels. However, the qualitative 405 behaviour of all dependences in the case of Newtonian and non-Newtonian liquids remains 406 similar. The time evolution of spreading radius of blood on time can be divided into two stages in 407 the case spreading/imbibition process of blood droplet over filter paper as in the case of 408 Newtonian liquids [19]. The two competing processes determine the whole spreading process: 409 the spreading of blood droplet, which results in an increase of the radius of the droplet base and 410 the imbibition of blood from droplet into the filter paper, which results in a shrinkage of the droplet 411 base. At the beginning, the base radius of droplet expanded to the maximum value. When the 412 droplet base radius started to decrease and the contact angle remained constant till complete 413 disappearance of the droplet. According to this two stage theory, the spreading behaviour of 414 blood and plasma show a similar behaviour. Two stages of the process prove that the spreading 415 of plasma and blood at all haematocrit levels are governed by a complete wetting of the filter 416 paper and in this case the spreading processes proceed in two stages [20]. Otherwise three 417 stages of the spreading/imbibition would exist [32]. 


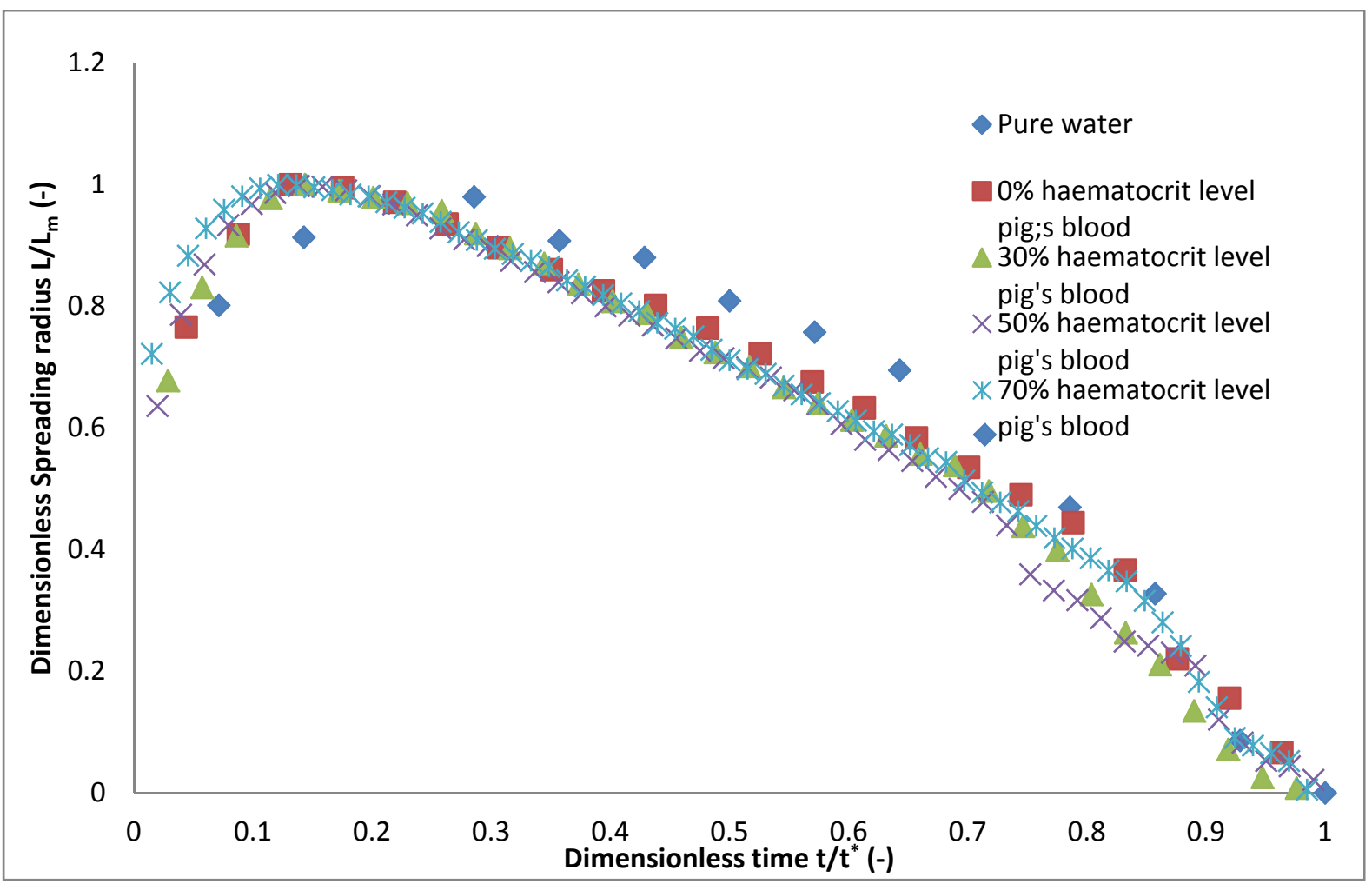

Figure 10. Dimensionless radii of the drop base as function of dimensionless time for water and blood samples at $0 \%, 30 \%, 50 \%$ and $70 \%$ haematocrit levels

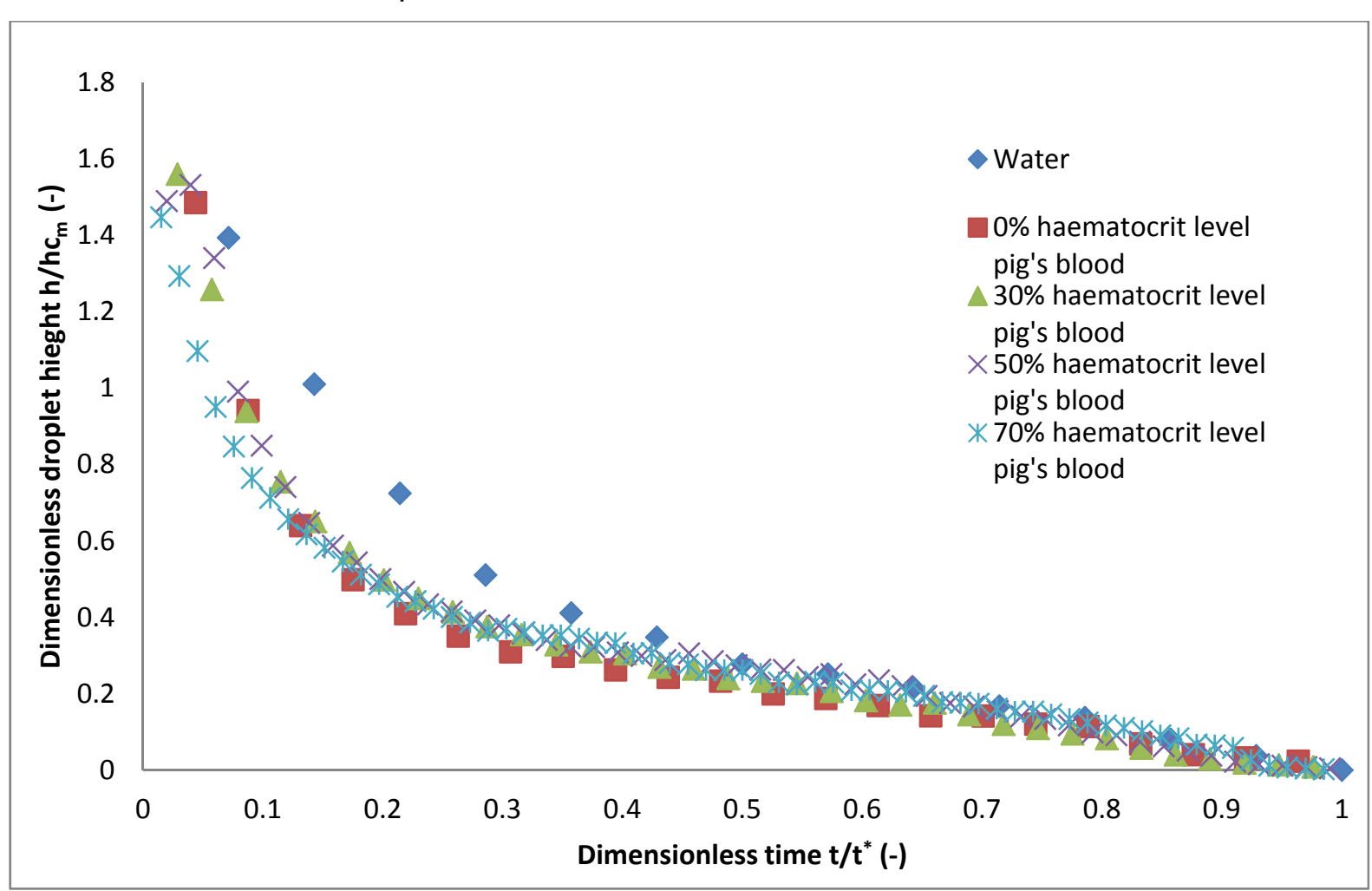

Figure 11. Dimensionless droplet heights of water, blood $0 \%, 30 \%, 50 \%$ and $70 \%$ haematocrit 


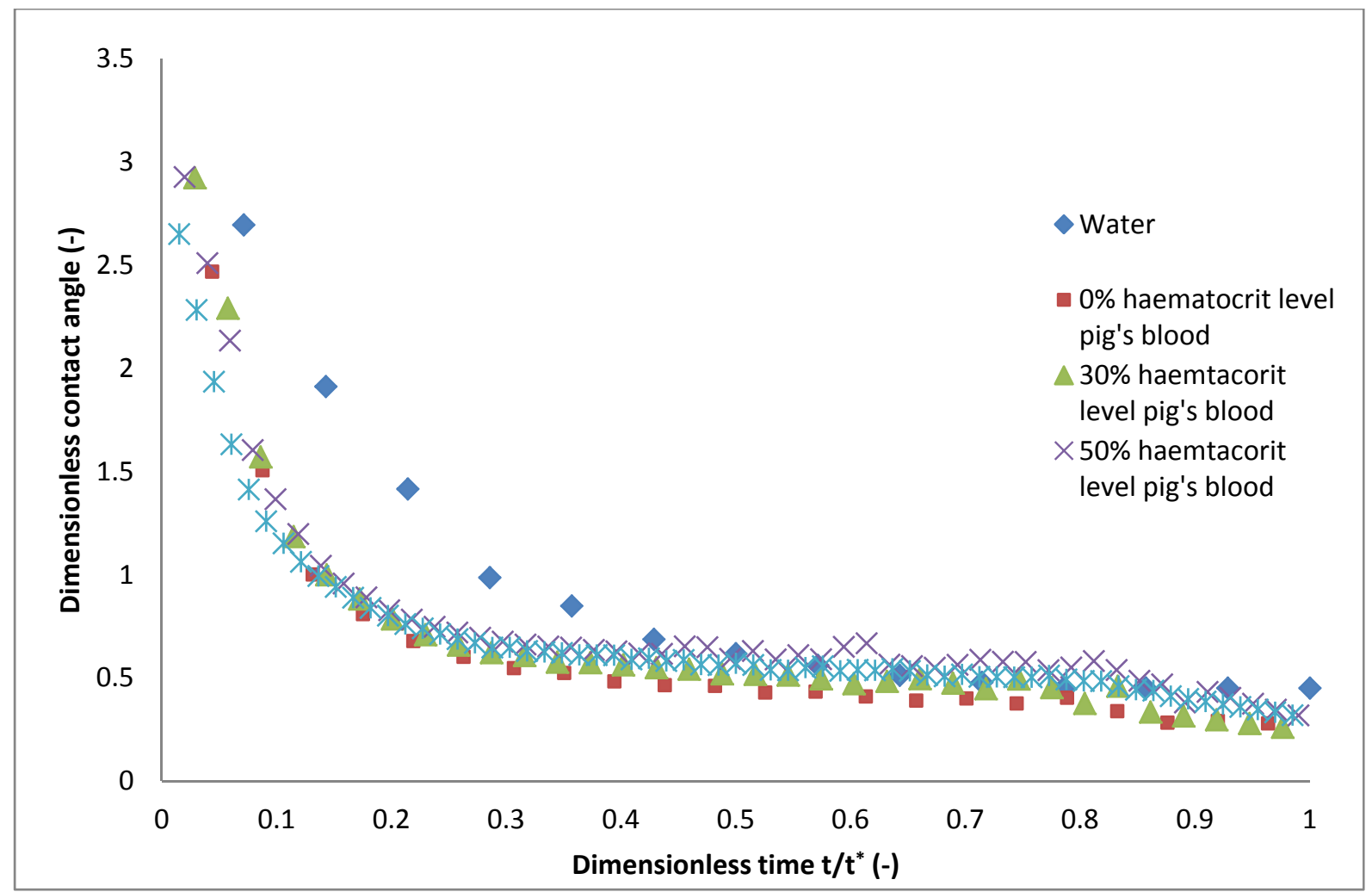

Figure 12. Dimensionless dynamic contact angles of water, blood plasma, $30 \%, 50 \%$ and $70 \%$

\section{Conclusion}

430 In this paper we have discussed the influence of different haematocrit levels of blood on the spreading/imbibition dynamics of DBS sampling on filter paper. For the droplet spreading over the filter paper, the size and spreading kinetic of blood samples on Whatman 903 filter paper has been demonstrated to decrease proportionally with the increase of haematocrit level in the blood. There is a significant decrease of wetting region as the presence of RBCs in blood plasma which indicates that the presence of RBCs in plasma-like solution considerably change the spreading/imbibition kinetics through porous fibre due to the shear-thinning behaviour of blood. The experimental data present the haematocrit effect on the spreading/imbibition dynamics of DBS sampling. The results suggest that all the spreading/imbibition dependences such as droplet height, droplet base radius and contact angle can be presented as universal functions of dimension less time. This behaviour of blood spreading allows us to control and calculate several spreading parameters of DBS sampling, such as, wetting region, retention volume, imbibition volume, liquid retention time above filter paper, etc. The development of a theoretical model based on the experimental data of spreading behaviour for determining the distribution of specific analytes is being considered and will be presented in our future work. 
1. Edelbroek, P. M.; Van der Heijden, J.; Stolk, L. M. L. Dried blood spot methods in therapeutic drug monitoring: methods, assays, and pitfalls. Therapeutic drug monitoring 2009, 31, 327-36.

2. Li, W.; Tse, F. L. S. Dried blood spot sampling in combination with LC-MS/MS for quantitative analysis of small molecules. Biomedical chromatography: BMC 2010, 24, 49-65.

3. Spooner, N.; Lad, R.; Barfield, M. Dried blood spots as a sample collection technique for the determination of pharmacokinetics in clinical studies: considerations for the validation of a quantitative bioanalytical method. Analytical chemistry 2009, 81, 1557-63.

4. Chaillet, P.; Zachariah, R.; Harries, K.; Rusanganwa, E.; Harries, a D. Dried blood spots are a useful tool for quality assurance of rapid HIV testing in Kigali, Rwanda. Transactions of the Royal Society of Tropical Medicine and Hygiene 2009, 103, 634-7.

5. Déglon, J.; Thomas, A.; Mangin, P.; Staub, C. Direct analysis of dried blood spots coupled with mass spectrometry: concepts and biomedical applications. Analytical and bioanalytical chemistry 2012, 402, 2485-98.

6. Barfield, M.; Spooner, N.; Lad, R.; Parry, S.; Fowles, S. Application of dried blood spots combined with HPLC-MS/MS for the quantification of acetaminophen in toxicokinetic studies. Journal of Chromatography B 2008, 870, 32-37.

7. Demirev, P. a Dried blood spots: analysis and applications. Analytical chemistry 2013, 85, 779-89.

8. Snijdewind, I. J. M.; Van Kampen, J. J. a; Fraaij, P. L. a; Van der Ende, M. E.; Osterhaus, A. D. M. E.; Gruters, R. a Current and future applications of dried blood spots in viral disease management. Antiviral research 2012, 93, 309-21.

9. Denniff, P.; Spooner, N. The effect of hematocrit on assay bias when using DBS samples for the quantitative bioanalysis of drugs. Bioanalysis 2010, 2, 1385-95.

10. Tanna, S.; Lawson, G. Analytical methods used in conjunction with dried blood spots.

11. Holub, M.; Tuschl, K.; Ratschmann, R.; Strnadova, K. A.; Muehl, A.; Heinze, G.; Sperl, W.; amino acids and acylcarnitines measured by tandem mass spectrometry. Clinica Chimica Acta 474 2006, 373, 27-31.

12. Keevil, B. G. The analysis of dried blood spot samples using liquid chromatography tandem mass spectrometry. Clinical biochemistry 2011, 44, 110-8.

477 13. Burnett, J. E. Dried blood spot sampling: practical considerations and recommendation for 478 use with preclinical studies. Bioanalysis 2011, 3, 1099-107.

479 14. Adam, B. W. J.; Alexander, R.; Smith, S. J.; Chace, D. H.; Loeber, J. G.; Elvers, L. H.; 
15. Peng, M.; Liu, L.; Peng, L. Evaluation of factors influencing accuracy in the analysis of succinylacetone in dried blood spots. Clinica Chimica Acta 2012, 413, 1265-1269.

16. Spooner, N.; Ramakrishnan, Y.; Barfield, M.; Dewit, O.; Miller, S. Use of DBS sample collection to determine circulating drug concentrations in clinical trials: practicalities and considerations. Bioanalysis 2010, 2, 1515-22.

17. Alleborn, N.; Raszillier, H. Spreading and sorption of a droplet on a porous substrate. Chemical Engineering Science 2004, 59, 2071-2088.

18. Kumar, S. M.; Deshpande, A. P. Dynamics of drop spreading on fibrous porous media. Colloids and Surfaces A: Physicochemical and Engineering Aspects 2006, 277, 157-163.

19. Starov, V. . M.; Zhdanov, S. A.; Kosvintsev, S. R.; Sobolev, V. D. Spreading of liquid drops over porous substrates. Advances in Colloid and Interface Science 2003, 104, 123-158.

20. Starov, V. M.; Kostvintsev, S. R.; Sobolev, V. D.; Velarde, M. G.; Zhdanov, S. a Spreading of liquid drops over dry porous layers: complete wetting case. Journal of colloid and interface science 2002, 252, 397-408. and Analysis of Human Whole Blood Specimens. The journal of nutrition 2001, 1631-1636.

22. Merrill, E. Rheology of blood. Physiol. Rev 1969, 49.

500

501

502

503

504

505

506

507

508

509

510

511

512

513

514

515

516

517

518

23. Baskurt, O. K.; Meiselman, H. J. Blood rheology and hemodynamics. Seminars in thrombosis and hemostasis 2003, 29, 435-50.

24. O'Mara, M.; Hudson-Curtis, B.; Olson, K.; Yueh, Y.; Dunn, J.; Spooner, N. The effect of hematocrit and punch location on assay bias during quantitative bioanalysis of dried blood spot samples. Bioanalysis 2011, 3, 2335-47.

25. O'Broin, S. Influence of Hematocrit on Quantitative Analysis of "Blood Spots" on Filter Paper. Clinical Chemistry 1993, 39, 1354-1355.

26. Hoogtanders, K.; Van der Heijden, J.; Christiaans, M.; Edelbroek, P.; Van Hooff, J. P.; Stolk, L. M. L. Therapeutic drug monitoring of tacrolimus with the dried blood spot method. Journal of pharmaceutical and biomedical analysis 2007, 44, 658-64.

27. Clarke, A.; Blake, T.; Carruthers, K.; Woodward, A. Spreading and imbibition of liquid droplets on porous surfaces. Langmuir 2002, 2980-2984.

28. Windberger, U.; Bartholovitsch, A.; Plasenzotti, R.; Korak, K. J.; Heinze, G. Whole blood viscosity, plasma viscosity and erythrocyte aggregation in nine mammalian species: reference values and comparison of data. Experimental physiology 2003, 88, 431-440.

29. Baskurt, O. K. Handbook of hemorheology and hemodynamics; Baskurt, O. K.; Hardeman, M. R.; Rampling, M. W.; Meiselman, H. J., Eds.; IOS Press, 2007; Vol. 69, pp. 1-455.

30. Thiriet, M. Biology and Mechanics of Blood Flows. Part I: Biology; Fieldman, J. S.; SaintAubin, Y.; Phong, D. H.; Vinet, L., Eds.; Springer, 2007; p. 652. 
519 31. Nassehi, V.; Das, D. B.; Shigidi, I. M. T. A.; Wakeman, R. J. Numerical analyses of bubble 520 point tests used for membrane characterisation : model development. Asia Pacific Journal of 521 Chemical Engineering Engineering 2011, 850-862.

522 32. Semenov, S.; Trybala, A.; Rubio, R. G.; Kovalchuk, N.; Starov, V.; Velarde, M. G. 523 Simultaneous spreading and evaporation: Recent developments. Advances in colloid and 524 interface science 2013.

525 